\title{
Angle-domain common-image gathers from anisotropic Gaussian beam migration and its application to anisotropy-induced imaging errors analysis
}

\author{
Jianguang $\mathrm{Han}^{1, *}$, Yun Wang ${ }^{2}$, Changqing $\mathrm{Yu}^{1}$ and Peng Chen ${ }^{3}$ \\ ${ }^{1}$ Institute of Geology, Chinese Academy of Geological Sciences, Beijing 100037, China. \\ ${ }^{2}$ School of Geophysics and Information Technology, China University of Geosciences, Beijing 100083, China. \\ ${ }^{3}$ State Key Laboratory of Oil and Gas Reservoir Geology and Exploitation, Chengdu University of Technology, \\ Chengdu 610059, China. \\ ${ }^{*}$ Corresponding author. e-mail: hanjianguang613@163.com
}

An approach for extracting angle-domain common-image gathers (ADCIGs) from anisotropic Gaussian beam prestack depth migration (GB-PSDM) is presented in this paper. The propagation angle is calculated in the process of migration using the real-value traveltime information of Gaussian beam. Based on the above, we further investigate the effects of anisotropy on GB-PSDM, where the corresponding ADCIGs are extracted to assess the quality of migration images. The test results of the VTI syncline model and the TTI thrust sheet model show that anisotropic parameters $\varepsilon, \delta$, and tilt angle $\theta$, have a great influence on the accuracy of the migrated image in anisotropic media, and ignoring any one of them will cause obvious imaging errors. The anisotropic GB-PSDM with the true anisotropic parameters can obtain more accurate seismic images of subsurface structures in anisotropic media.

\section{Introduction}

The earth is anisotropic in nature, and many sedimentary rocks exhibit seismic anisotropy (Crampin et al. 1984; Wang 2002; Wang et al. 2015). Seismic waves are affected by anisotropy, so ignoring anisotropy in migration may lead to obvious imaging errors (Larner and Cohen 1993; Alkhalifah and Larner 1994; Etgen et al. 2009). Migration imaging algorithms in the presence of anisotropy have been presented in a number of studies. Sena and Toksoz (1993) extended the isotropic Kirchhoff depth migration scheme to the anisotropic media by using anisotropic ray tracing. Tong et al. (1998) studied the true amplitude Kirchhoff migration technique in anisotropic media. Ferguson and Margrave (1998) addressed non-stationary phase shift method for transversely isotropic (TI) media. Ristow (1999) developed a depth migration scheme for TI media with a vertical symmetry axis (VTI) based on implicit finite-difference operators. Baumstein and Anderson (2003) combined the phase-shift and explicit correction operators to perform wavefield extrapolation in VTI media. Pedersen et al. (2010) derived phase slowness expressions for $\mathrm{P}$ - and $\mathrm{SV}$-waves that are used in a one-way wave-equation migration scheme in VTI media. In addition, many studies about anisotropic reverse time migration (RTM) have been proposed (Du et al. 2007; Fletcher et al. 2009; Fowler et al. 2010; Duveneck and Bakker 2011; Zhang et al. 2011; Liu et al. 2012; Zhan et al. 2012).

Gaussian beam migration is an excellent and efficient imaging method for processing seismic data because of its imaging accuracy comparable

Keywords. Gaussian beam migration; anisotropy; angle-domain common-image gathers; numerical analysis. 
to that of wave-equation migration and flexibility comparable to that of Kirchhoff migration. It can naturally collect energy from multipath contributions to solve for multi-valued travel times with different superposed beams. The Gaussian beam migration method was first presented in Hill (1990) for poststack applications, and later extended to prestack data (Hill 2001). Since then, several studies of Gaussian beam migration in isotropic media have been published (Gray 2005; Gray and Bleistein 2009; Popov et al. 2010; Yue et al. 2010; Han et al. 2013, 2014a). Meanwhile, Gaussian beam migration in anisotropic media was studied. The first extension to the anisotropic media was developed by Alkhalifah (1995), where Gaussian beam migration is performed for poststack data. Zhu et al. (2007) proposed a prestack Gaussian beam depth migration method in anisotropic media. Han et al. (2014b) presented a converted wave Gaussian beam migration method for TI media and applied it with encouraging results to synthetic data. Protasov (2015) presented a true-amplitude Gaussian beam imaging method of multicomponent seismic data in anisotropic elastic media.

Angle-domain common-image gathers (ADCIGs) have become a common tool for analysing prestack depth migration images. Several researchers have studied the extraction scheme of ADCIGs by using Kirchhoff migration methods (Operto et al. 2000; Rousseau et al. 2000; Xu et al. 2001; BrandsbergDahl et al. 2003; Koren et al. 2008) and wave-equation migration methods (de Bruin et al. 1990; Rickett and Sava 2002; Sava and Fomel 2003; Biondi and Symes 2004; Rosales et al. 2008; Zhang et al. 2010; Sava and Vlad 2011; Xu et al. 2011; Zhang and McMechan 2011). The problems observed for offset-domain common-image gathers (ODCIGs), such as failing to properly characterise complex propagation paths and producing artifacts because of the ambiguity of reflector positions caused by multipathing, can be alleviated using ADCIGs. Unlike ODCIGs, the ADCIGs use the subsurface angle as the index instead of the offset, which are less susceptible to multiple paths effects than ODCIGs (Sava and Fomel 2003). The moveout of the ADCIGs carries the traveltime errors for waves propagating in different angles through the velocity model (Yan and Xie 2012). For the correct migration velocity model, the events on the ADCIGs are flat and correctly positioned at depth. Migrating with an incorrect velocity model, however, leads to inconsistent angle-domain reflectivity and generates residual curvature in the ADCIG volume.

In this paper, we describe an approach for computing ADCIGs in conjunction with anisotropic Gaussian beam migration and apply it to discussing the effects of anisotropy on imaging. We first introduce the principle of extracting ADCIGs from anisotropic Gaussian beam prestack depth migration (GB-PSDM), where the propagation angle is calculated in the process of migration using the real-value travel time information of Gaussian beam. Then we perform a numerical model analysis to investigate the effects of anisotropic parameters $\varepsilon, \delta$ and tilt angle $\theta$ on GB-PSDM, where the corresponding ADCIGs are used to assess the quality of migration images.

\section{GB-PSDM theory in anisotropic media}

The image of GB-PSDM is computed by crosscorrelating the downward-continued recorded wavefield and the forward-modelled source wavefield. The common shot gathers GB-PSDM formula in two-dimensional media can be written as (Han and Wang 2015)

$$
\begin{aligned}
I(\mathbf{x})= & C \int d x_{s} \sum_{\mathbf{L}_{r}} \int d \omega \int d p_{s x} \int d p_{r x} D\left(\mathbf{L}_{r}, \mathbf{p}_{r}, \omega\right) \\
& \times u_{G B}^{*}\left(\mathbf{x}, \mathbf{x}_{s}, \mathbf{p}_{s}, \omega\right) u_{G B}^{*}\left(\mathbf{x}, \mathbf{L}_{r}, \mathbf{p}_{r}, \omega\right),
\end{aligned}
$$

where $\mathbf{x}_{s}$ and $\mathbf{x}_{r}$ denote the source and receiver locations. $I(\mathbf{x})$ is the final image at subsurface point $\mathbf{x}, \omega$ is circular frequency, and $C$ denotes corresponding constant. $u_{G B}^{*}\left(\mathbf{x}, \mathbf{x}_{s}, \mathbf{p}_{s}, \omega\right)$ and $u_{G B}^{*}\left(\mathbf{x}, \mathbf{L}_{r}, \mathbf{p}_{r}, \omega\right)$ are the complex conjugates of the normalised Gaussian beam solutions to the wave equation, where the former represents that wave propagates from source $\mathbf{x}_{s}$ with initial direction $\mathbf{p}_{s}$, and the latter signifies that wave propagates from the beam center $\mathbf{L}_{r}$ with initial direction $\mathbf{p}_{r} . D\left(\mathbf{L}_{r}, \mathbf{p}_{r}, \omega\right)$ is the local plane wave component obtained from a local slant stack of the commonshot traces (Gray and Bleistein 2009). The local slant stack is the principal algorithm in the Gaussian beam migration method, which decomposes a certain range of seismic records near the beam center into different outgoing directional local plane waves for wave-field extrapolation.

The shape of the Gaussian beam in the anisotropic media is built as it would be done in the isotropic case, but along anisotropic direction of propagation (Červený and Pšenčík 2010). If functions $A$ and $T$ are used to represent complex amplitude and traveltime, respectively, of the Gaussian beam, then the Gaussian beam expression can be written as

$$
u_{G B}\left(\mathbf{x}, \mathbf{x}_{\mathbf{0}}, \mathbf{p}, \omega\right)=A \exp (i \omega T) .
$$

The extension of Gaussian beam migration to anisotropic media requires calculation of the complex-valued time and amplitude using anisotropic kinematic and dynamic ray tracing, which is the key of the anisotropic GB-PSDM. The kinematic 
ray tracing system for general inhomogeneous anisotropic media is given by Červený $(1972,2001)$ :

$$
\frac{d x_{i}}{d \tau}=a_{i j k l} p_{l} g_{j} g_{k}
$$

and

$$
\frac{d p_{i}}{d \tau}=-\frac{1}{2} \frac{\partial a_{j k l n}}{\partial x_{i}} p_{k} p_{n} g_{j} g_{l}
$$

where $\tau$ is the traveltime along the ray, $p_{i}$ are the components of the slowness vector, $a_{i j k l}$ are the density-normalised elastic parameters, and $g_{j}$ are the components of the eigenvector for the Christoffel matrix $\boldsymbol{\Gamma}$.

In two dimensions along a symmetry plane, all components with subscript 2 are eliminated. The anisotropic ray tracing system (3) can be written as:

$$
\begin{aligned}
\frac{d x_{1}}{d \tau}= & a_{11} p_{1} g_{1} g_{1}+a_{15} p_{3} g_{1} g_{1}+2 a_{15} p_{1} g_{1} g_{3}+a_{13} p_{3} g_{1} g_{3} \\
& +a_{55} p_{3} g_{1} g_{3}+a_{55} p_{1} g_{3} g_{3}+a_{35} p_{3} g_{3} g_{3},
\end{aligned}
$$

$$
\begin{aligned}
\frac{d x_{3}}{d \tau}= & a_{15} p_{1} g_{1} g_{1}+a_{55} p_{3} g_{1} g_{1}+a_{55} p_{1} g_{1} g_{3}+2 a_{35} p_{3} g_{1} g_{3} \\
& +a_{13} p_{1} g_{1} g_{3}+a_{35} p_{1} g_{3} g_{3}+a_{33} p_{3} g_{3} g_{3}, \quad(4 \mathrm{~b})
\end{aligned}
$$

$$
\begin{aligned}
\frac{d p_{1}}{d \tau}= & -\frac{1}{2}\left(\frac{\partial a_{11}}{\partial x_{1}} p_{1} p_{1} g_{1} g_{1}+2 \frac{\partial a_{15}}{\partial x_{1}} p_{1} p_{3} g_{1} g_{1}\right. \\
& +2 \frac{\partial a_{15}}{\partial x_{1}} p_{1} p_{1} g_{1} g_{3}+2 \frac{\partial a_{13}}{\partial x_{1}} p_{1} p_{3} g_{1} g_{3} \\
& +2 \frac{\partial a_{55}}{\partial x_{1}} p_{1} p_{3} g_{1} g_{3}+\frac{\partial a_{55}}{\partial x_{1}} p_{1} p_{1} g_{3} g_{3} \\
& +2 \frac{\partial a_{35}}{\partial x_{1}} p_{1} p_{3} g_{3} g_{3}+\frac{\partial a_{55}}{\partial x_{1}} p_{3} p_{3} g_{1} g_{1} \\
& \left.+2 \frac{\partial a_{35}}{\partial x_{1}} p_{3} p_{3} g_{1} g_{3}+\frac{\partial a_{33}}{\partial x_{1}} p_{3} p_{3} g_{3} g_{3}\right)
\end{aligned}
$$

and

$$
\begin{aligned}
\frac{d p_{3}}{d \tau}= & -\frac{1}{2}\left(\frac{\partial a_{11}}{\partial x_{3}} p_{1} p_{1} g_{1} g_{1}+2 \frac{\partial a_{15}}{\partial x_{3}} p_{1} p_{3} g_{1} g_{1}\right. \\
& +2 \frac{\partial a_{15}}{\partial x_{3}} p_{1} p_{1} g_{1} g_{3}+2 \frac{\partial a_{13}}{\partial x_{3}} p_{1} p_{3} g_{1} g_{3} \\
& +2 \frac{\partial a_{55}}{\partial x_{3}} p_{1} p_{3} g_{1} g_{3}+\frac{\partial a_{55}}{\partial x_{3}} p_{1} p_{1} g_{3} g_{3} \\
& +2 \frac{\partial a_{35}}{\partial x_{3}} p_{1} p_{3} g_{3} g_{3}+\frac{\partial a_{55}}{\partial x_{3}} p_{3} p_{3} g_{1} g_{1} \\
& \left.+2 \frac{\partial a_{35}}{\partial x_{3}} p_{3} p_{3} g_{1} g_{3}+\frac{\partial a_{33}}{\partial x_{3}} p_{3} p_{3} g_{3} g_{3}\right) .
\end{aligned}
$$

The ray tracing system (4) requires the medium to be specified in terms of elastic parameters. However, the anisotropy for the most commonly used in TI media is usually described with Thomsen parameters, which facilitate the research of the anisotropic media. According to the relationship between the Thomsen parameters and the elastic constants (Thomsen 1986), the density-normalised elastic constants of the VTI media can be expressed using the Thomsen parameters as:

$a_{11}=(1+2 \varepsilon) V_{P 0}^{2}, \quad a_{33}=V_{P 0}^{2}, \quad a_{55}=V_{S 0}^{2}$,

$a_{13}=\sqrt{2 \delta a_{33}\left(a_{33}-a_{55}\right)+\left(a_{33}-a_{55}\right)^{2}}-a_{55}$

where $V_{P 0}$ and $V_{S 0}$ are the phase velocities of Pand SV-waves along the symmetry axis of VTI media, respectively; $\varepsilon$ and $\delta$ are the anisotropic parameters that characterise the magnitude of the anisotropy. $\gamma$ is not considered because it determines the anisotropy of the $\mathrm{SH}$-wave, which is not discussed in this paper. The elastic constants of TI media with a tilted symmetry axis (TTI) can be obtained from the ones of the VTI media via the Bond transformation (Winterstein 1990).

\section{ADCIGs from anisotropic Gaussian beam migration}

The propagation angle (between the wavefield propagation direction and positive direction of $Z$ axis) can be obtained in the process of anisotropic Gaussian beam migration using the real-value traveltime information of Gaussian beam, and then the incidence angle (between the source wavefield propagation direction and the reflector normal) can be computed, which can be used to extract ADCIGs. In two-dimensional ray centered coordinate system, as shown in figure 1 , the real-value traveltime of Gaussian beam at the point $R$ in the anisotropic media can be written as:

$$
\tau=\tau(Q)+\frac{1}{2} \operatorname{Re}\left[\frac{\mathbf{p}(Q)}{\mathbf{q}(Q)}\right] n^{2} .
$$

This is the same equation used for the isotropic case. However, $\tau(Q)$ is the travel-time at the point $Q$ obtained from anisotropic kinematic ray tracing,

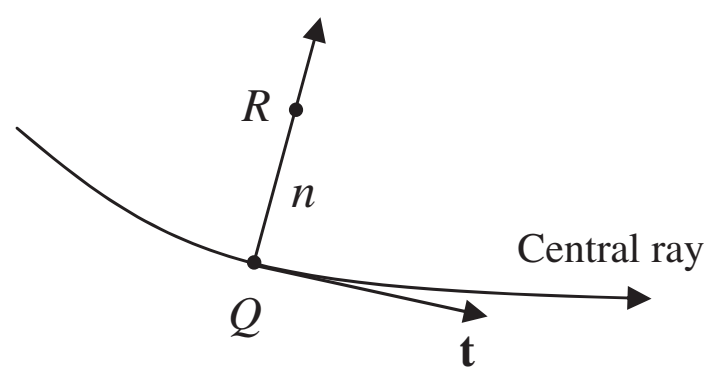

Figure 1. Ray centered coordinate system. $n$ is distance normal from the point $R$ to the point $Q$ on the central ray. t represents the phase velocity direction at the point $Q$. 
and $n$ is the distance normal to the phase velocity direction. Furthermore, $\mathbf{p}(Q)$ and $\mathbf{q}(Q)$ are computed using the anisotropic dynamic ray tracing equations given by Hanyga (1986) and Alkhalifah (1995).

The expression (6) in Cartesian coordinate system can be rewritten as:

$$
\tau=\tau(Q)+\frac{1}{2} \operatorname{Re}\left[\frac{\mathbf{p}(Q)}{\mathbf{q}(Q)}\right]\left[\left(x-x_{Q}\right)^{2}+\left(z-z_{Q}\right)^{2}\right] .
$$

Taking the derivative of both sides of the equation (7) with respect to $x$ and $z$, respectively, we can obtain

$$
p_{x}=p_{x}(Q)+\left(x-x_{Q}\right) \operatorname{Re}\left[\frac{\mathbf{p}(Q)}{\mathbf{q}(Q)}\right]
$$

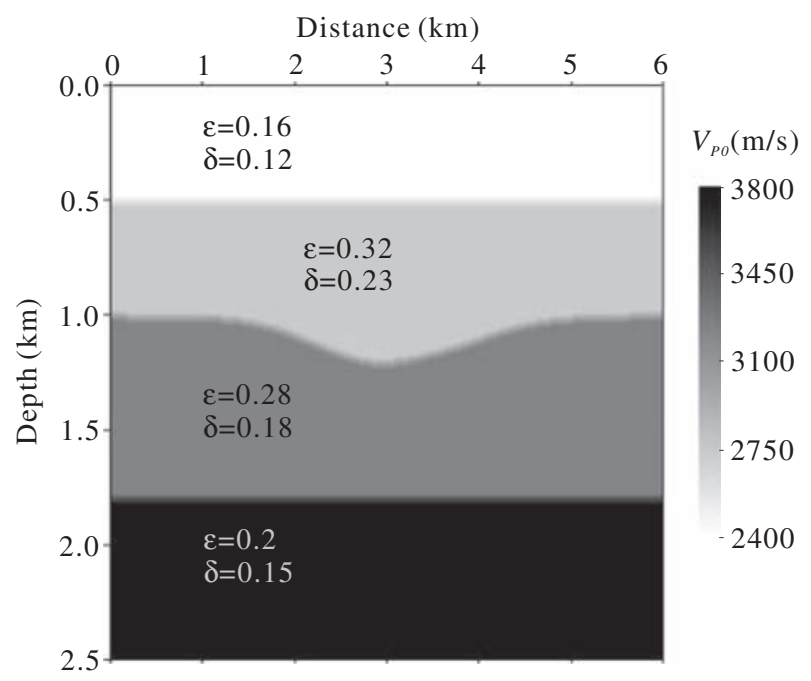

Figure 2. Syncline model with different anisotropy parameters in each layer.

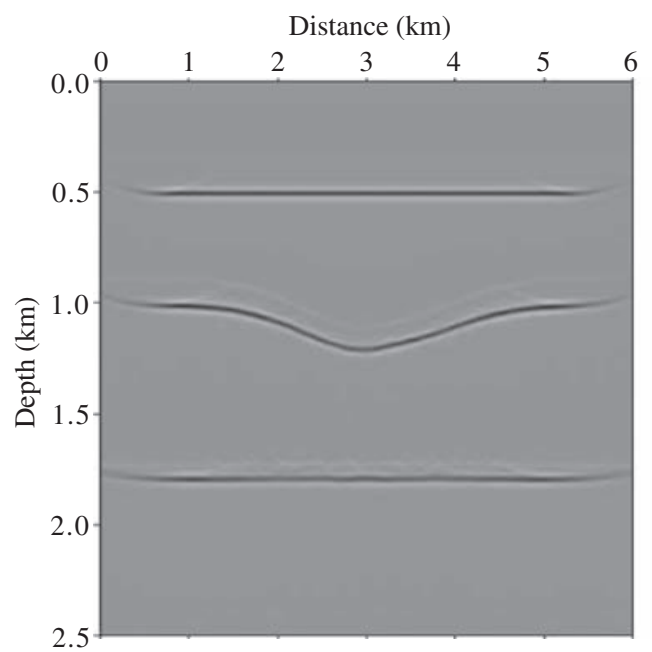

(a) and

$$
p_{z}=p_{z}(Q)+\left(z-z_{Q}\right) \operatorname{Re}\left[\frac{\mathbf{p}(Q)}{\mathbf{q}(Q)}\right],
$$

where $p_{x}$ and $p_{z}$ represent the horizontal and vertical components of the slowness vector, respectively, and they can also be expressed as:

$$
p_{x}=\frac{\sin \varphi}{v}, \quad p_{z}=\frac{\cos \varphi}{v} .
$$

where $\varphi$ is the propagation angle and $v$ is the phase velocity. Taking into account equations (8 and 9 ), we can calculate the propagation angle at grid point $R$ as follows:

$$
\tan \varphi=\frac{p_{x}(Q)+\left(x-x_{Q}\right) \operatorname{Re}\left[\frac{\mathbf{p}(Q)}{\mathbf{q}(Q)}\right]}{p_{z}(Q)+\left(z-z_{Q}\right) \operatorname{Re}\left[\frac{\mathbf{p}(Q)}{\mathbf{q}(Q)}\right]} .
$$

After the propagation angles $\varphi_{s}$ and $\varphi_{r}$ are computed with the Gaussian beams coming from the source and receiver, respectively, the aperture angle can be obtained. The incidence angle is half of the aperture angle for the PP-wave. And then the ADCIGs can be extracted by the incidence angle computed in the process of Gaussian beam migration.

\section{Numerical model analysis}

\subsection{Syncline model}

In this section, a VTI syncline model is used to examine the effect of anisotropic parameters $\varepsilon$ and $\delta$ on Gaussian beam prestack depth migration. The model contains four layers with different anisotropic parameters in each layer, as shown in

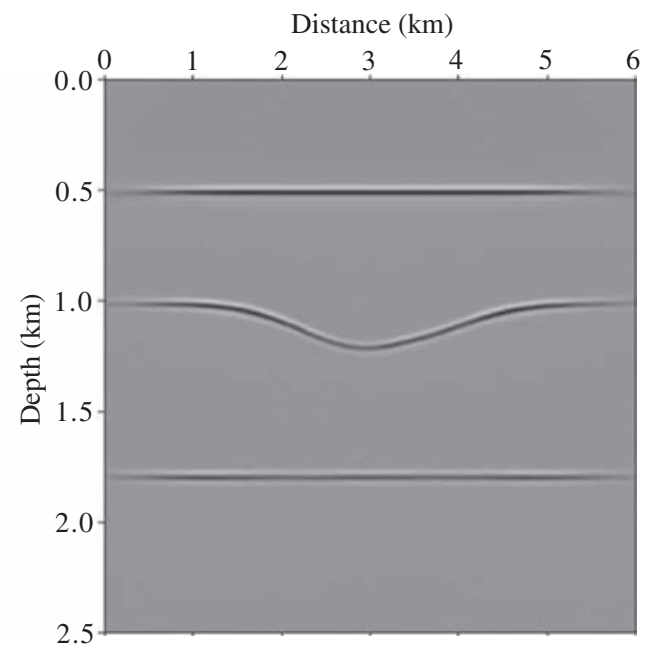

(b)

Figure 3. Migration results of syncline model using (a) isotropic GB-PSDM and (b) anisotropic GB-PSDM. Anisotropic migration provides a correct image, and the migration result exactly matches the true interfaces. 


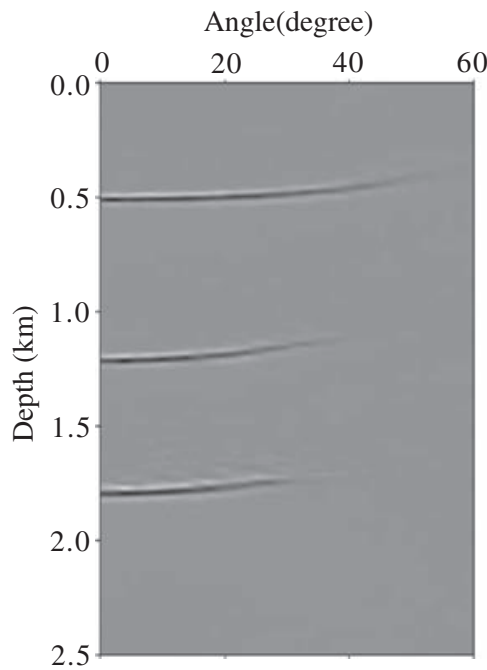

(a)

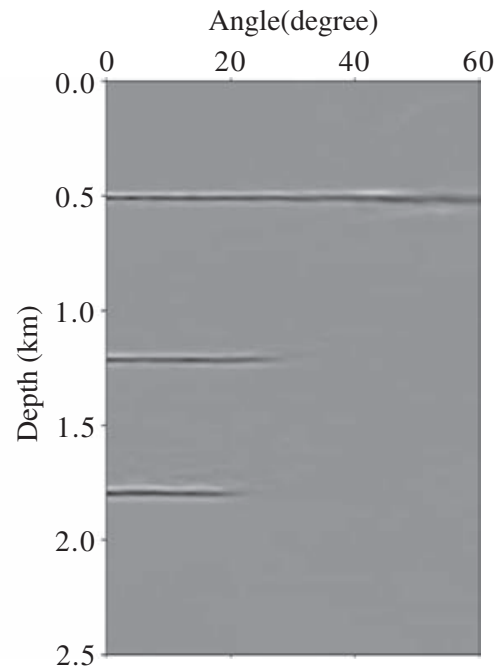

(b)

Figure 4. ADCIGs for a surface location at $3000 \mathrm{~m}$ from (a) isotropic GB-PSDM and (b) anisotropic GB-PSDM.

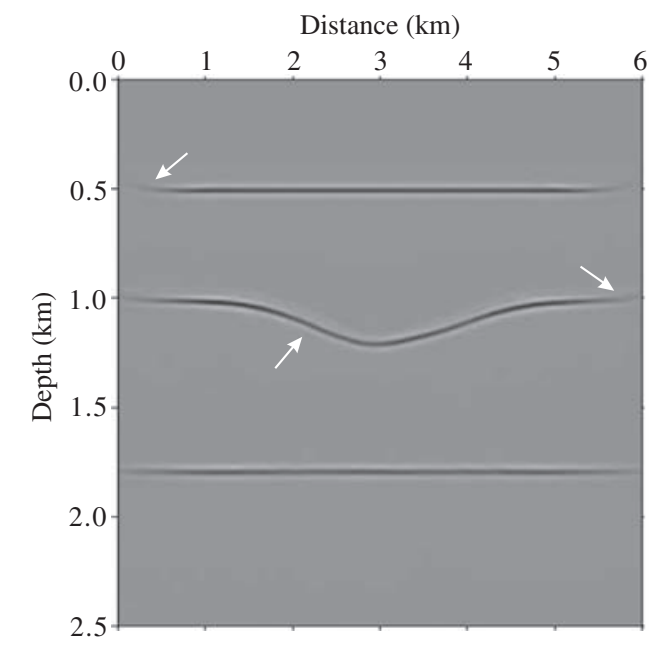

(a)

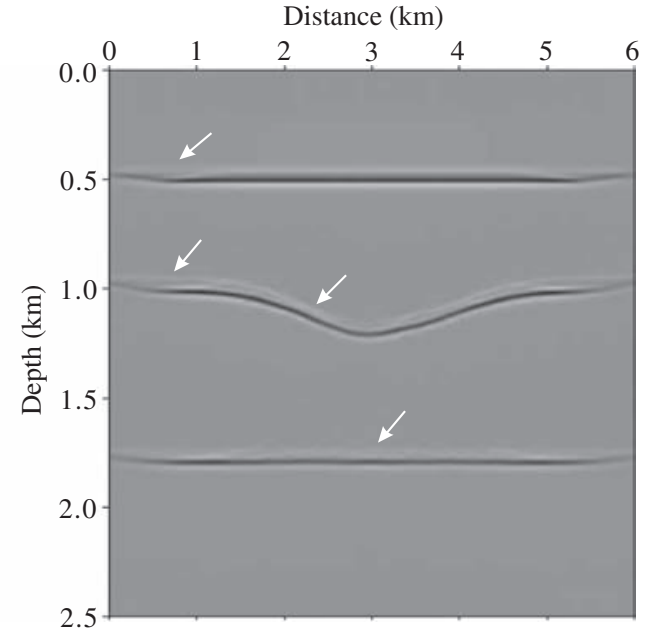

(b)

Figure 5. Migration results using the anisotropic GB-PSDM with the anisotropic parameter $(\mathbf{a}) \varepsilon=0$ and $(\mathbf{b}) \delta=0$.

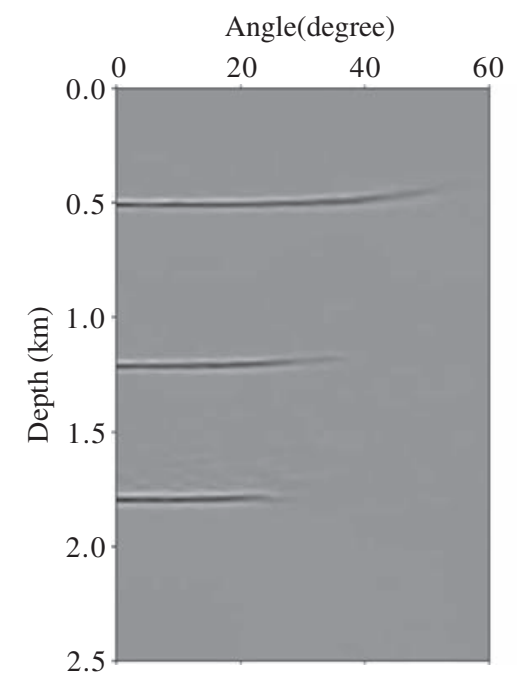

(a)
Angle(degree)

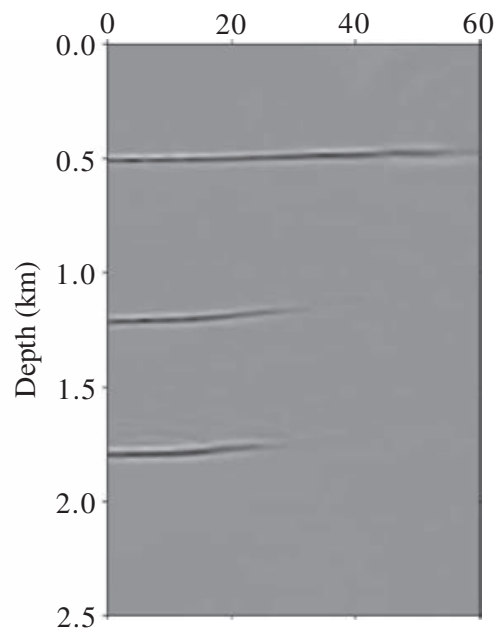

(b)

Figure 6. ADCIGs for a surface location at $3000 \mathrm{~m}$ from the anisotropic GB-PSDM with the anisotropic parameter $(\mathbf{a}) \varepsilon=0$ and (b) $\delta=0$. 
figure 2. Synthetic data generated by anisotropic ray tracing forward modelling method consist of 121 shot gathers with shot interval of $40 \mathrm{~m}$. The source wavelet is the Ricker wavelet with a dominant frequency of $30 \mathrm{~Hz}$. There are 321 receivers per shot, and the receiver spacing is $10 \mathrm{~m}$. The traveltime is $1.8 \mathrm{~s}$ with $2 \mathrm{~ms}$ of sampling.

Figure 3(a and $\mathrm{b})$ displays the resulting images using isotropic and anisotropic GB-PSDM, respectively. With the velocity $V_{P}=V_{P 0}$, the isotropic GB-PSDM result (figure 3a) shows that the image is not accurate and the noise is serious near the reflection interfaces. The image obtained using anisotropic GB-PSDM with the true model parameters (figure $3 \mathrm{~b}$ ) is found to be an excellent match with the exact model interfaces, and the defocusing artifacts are eliminated. The differences between the isotropic and anisotropic GB-PSDM are evident by looking at the ADCIGs. Figure 4 compares ADCIGs located at $3000 \mathrm{~m}$ obtained from the isotropic and anisotropic GB-PSDM. The ADCIGs extracted from the anisotropic GB-PSDM (figure 4b) are flat, while we notice significant residuals in the isotropic ADCIGs.

To further examine the effects of anisotropic parameters $\varepsilon$ and $\delta$ on the migration imaging, the anisotropic GB-PSDM is performed with the anisotropic parameter $\varepsilon=0$ and $\delta=0$, respectively. The migration results are shown in figure 5, and the corresponding ADCIGs are shown in figure 6. Although, the defocusing artifacts are not obvious in figure 5(a), the image is not accurate, especially for the curved interface of the synclinal structure and the edge position of model as shown by the arrow in figure $5(\mathrm{a})$. The events in the ADCIGs obtained from the anisotropic GB-PSDM with the anisotropic parameter $\varepsilon=0$ (figure 6a)

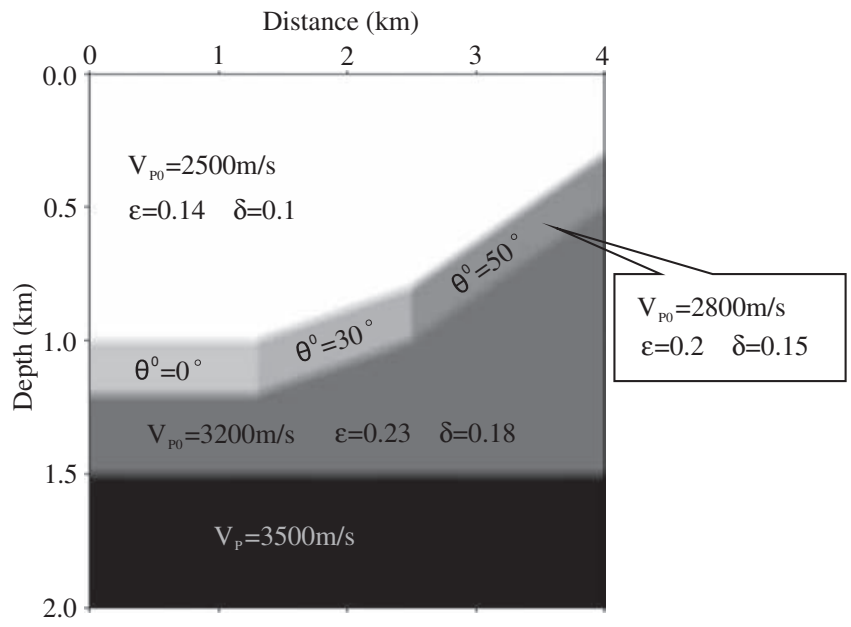

Figure 7. Subsurface TTI media model including a TTI thrust sheet and a flat reflector. The thrust sheet is composed of three blocks in the model with a spatially varying symmetry axis.

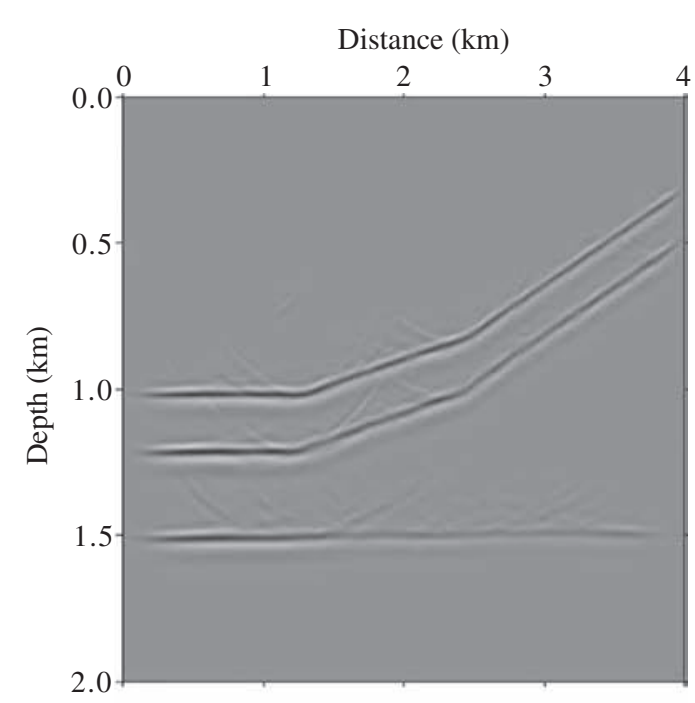

(a)

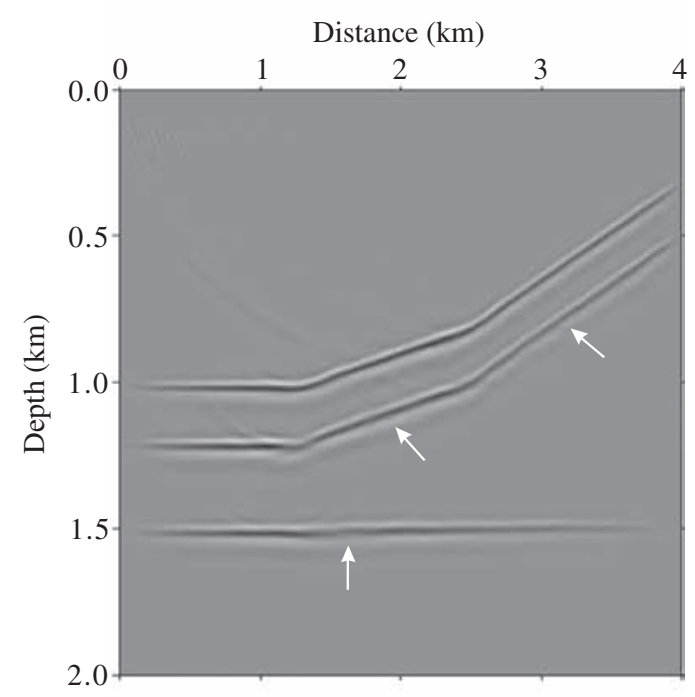

(b)

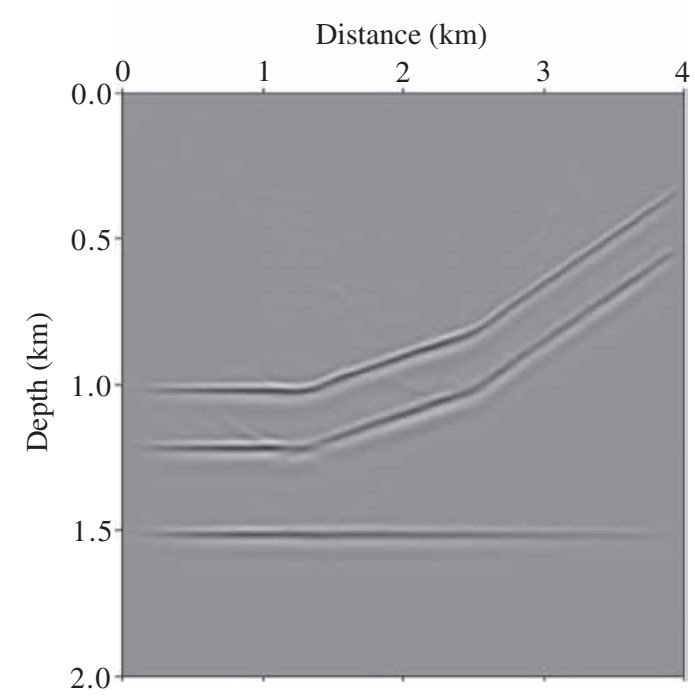

(c)

Figure 8. Migration results of thrust sheet model using (a) isotropic, (b) VTI and (c) TTI GB-PSDM. 


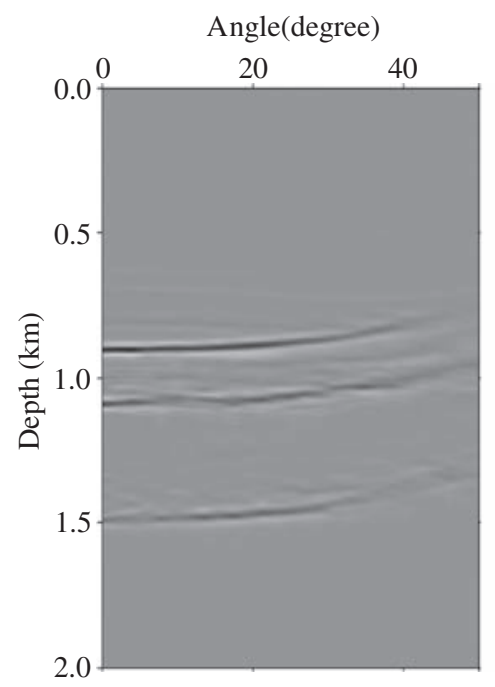

(a)

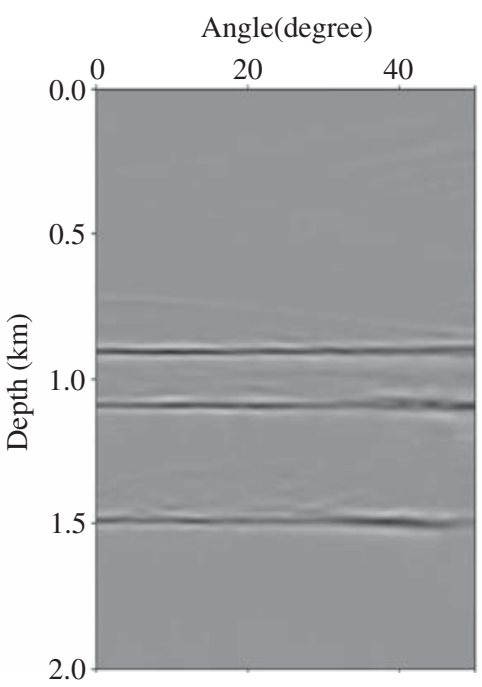

(b)

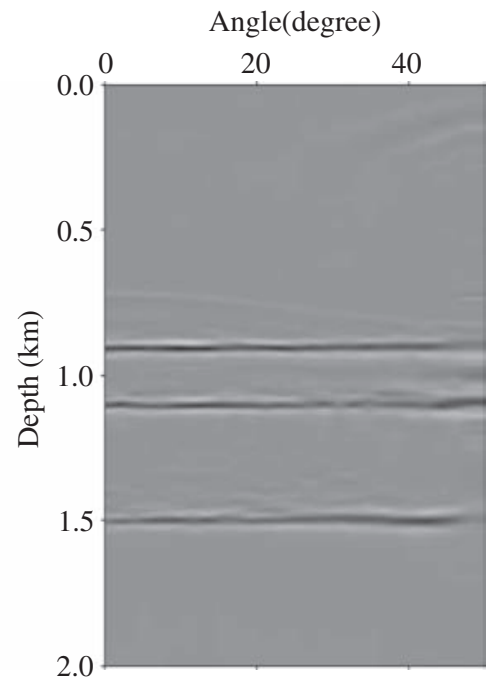

(c)

Figure 9. ADCIGs for a surface location at $1900 \mathrm{~m}$ from (a) isotropic, (b) VTI and (c) TTI GB-PSDM.

apparently curve up at large incidence angles. By contrast, imaging errors using anisotropic GBPSDM with the anisotropic parameter $\delta=0$ are more obvious, and the noise is significant near the reflection interfaces, as indicated by the arrow in figure 5(b). Moreover, the ADCIGs in figure 6(b) are not flat. The test results demonstrate that anisotropy has a great influence on GB-PSDM in VTI media, and ignoring anisotropy in migration may lead to obvious imaging errors. Therefore, in addition to accurate migration velocity model, the true anisotropic parameters $\varepsilon$ and $\delta$ are essential for migration imaging in VTI media.

\subsection{Thrust sheet model}

Next, a TTI thrust sheet model is used to investigate the influence of the tilt angle of the symmetry axes $\theta$ on GB-PSDM. The model contains a TTI thrust sheet and a flat reflector as shown in figure 7 . The first and third layers are VTI media with different anisotropic parameters. The thrust sheet is composed of three blocks in the model, and each block has a unique axis of symmetry. Synthetic dataset is generated using the anisotropic ray tracing forward modeling method, and the source wavelet is the Ricker wavelet with a dominant frequency of $30 \mathrm{~Hz}$. A total of 77 shots are acquired on the surface with 401 receivers per shot. The shot spacing is $50 \mathrm{~m}$, and the receiver spacing is $10 \mathrm{~m}$. The travel-time is $2 \mathrm{~s}$, with $2 \mathrm{~ms}$ of sampling.

Figure 8(a) displays the resulting image using the isotropic GB-PSDM with the velocity $V_{P}=$ $V_{P 0}$, where the flat reflector beneath the thrust sheet exhibits a substantial pull-up and the energy cannot be focused. The dipping interface of the thrust sheet is not imaged at the true position, and the defocusing artifacts are noticeable near the reflection interfaces. Figure $8(\mathrm{~b})$ shows the image using the anisotropic GB-PSDM when $\varepsilon, \delta$ and $V_{P 0}$ are correct, but the tilt angle $\theta$ in the thrust sheet is not considered. Although, the image of the reflectors is improved and the defocusing artifacts are eliminated, there are still mispositioning problems. The flat reflector beneath the thrust sheet still exhibits a spurious pull-up and the lower flank of the thrust sheet is incorrectly imaged, as indicated by the arrow in figure 8(b). In contrast, the image from anisotropic GB-PSDM with the true model parameters (figure 8c) shows that the flat reflector is well focused and positioned. The image of the thrust sheet matches very well with the correct position in the model, and the defocusing artifacts around it are also eliminated. To assess the quality of migration images, we examine ADCIGs for a location at $1900 \mathrm{~m}$ (figure 9). In the isotropic ADCIGs (figure 9a), the events are mispositioned and they are not flat. The VTI ADCIGs (figure 9b) have some nearly flat events. However, they are shif ted upward compared with the TTI ADCIGs (figure 9c). The events in the TTI ADCIGs (figure 9c) are flat and produce the correct image. The test results show that the tilt angle $\theta$ has a significant influence on the accuracy of migrated image and the correct tilt angle is also essential for migration imaging in TTI media.

\section{Conclusions}

We have presented an approach for extracting ADCIGs from anisotropic GB-PSDM based on anisotropic kinematic and dynamic ray tracing 
systems, where the propagation angle is calculated in the process of anisotropic Gaussian beam migration using the real-value traveltime information of Gaussian beam. In addition, we have discussed the effects of each of anisotropic parameters on GB-PSDM by using the ADCIGs. The test results of the VTI syncline model show that anisotropic parameters $\varepsilon$ and $\delta$ have a great influence on GB-PSDM in VTI media, and ignoring any one of them in migration will lead to obvious imaging errors. Moreover, tests of the TTI thrust sheet model show that the tilt angle $\theta$ also has a significant influence on the accuracy of the migrated image in TTI media. Although, the image quality using the VTI GB-PSDM is improved compared with the isotropic GB-PSDM, there are still mispositioning problems because of the ignoring of the tilt angle. Therefore, in addition to accurate migration velocity model, the true anisotropic parameters $\varepsilon$ and $\delta$ are essential for migration imaging in VTI media, and the correct tilt angle $\theta$ is also essential for migration imaging in TTI media. The anisotropic GB-PSDM taking the influence of anisotropy on seismic waves into account can obtain accurate seismic images of subsurface structures in anisotropic media.

\section{Acknowledgements}

The research is supported by the Fundamental Research Funds for Central Public Welfare Research Institutes, CAGS (no. J1620), China Postdoctoral Science Foundation (no. 2016M591250) and the National Natural Science Foundation of China (nos. 41425017, 41374131, 41374128).

\section{References}

Alkhalifah T 1995 Gaussian beam depth migration for anisotropic media; Geophysics 60 1474-1484.

Alkhalifah T and Larner K 1994 Migration error in transversely isotropic media; Geophysics 59 1405-1418, doi: 10.1190/1.1443698.

Baumstein A and Anderson J 2003 Wavefield extrapolation in laterally varying VTI media; 73th Annual International Meeting, SEG, Expanded Abstracts 945-948.

Biondi B and Symes W 2004 Angle-domain commonimage gathers for migration velocity analysis by wavefield continuation imaging; Geophysics 69 1283-1298, doi: 10.1190/1.1801945.

Brandsberg-Dahl S, De Hoop M V and Ursin B 2003 Focusing in dip and AVA compensation on scatteringangle/azimuth common-image gathers; Geophysics 68 232-254, doi: 10.1190/1.1543210.

Červený V 1972 Seismic rays and ray intensities in inhomogeneous anisotropic media; Geophys. J. Roy. Astr. Soc. 29 1-13, doi: 10.1111/j.1365-246X.1972.tb06147.x.

Červený V 2001 Seismic ray theory; Cambridge University Press, Cambridge.
Červený V and Pšenčík I 2010 Gaussian beams in inhomogeneous anisotropic layered structures; Geophys. J. Int. $180798-812$.

Crampin S, Chesnokov E M and Hipkin R G 1984 Seismic anisotropy - the state-of-the art: II; Geophys. J. Roy. Astr. Soc. 76 1-16, doi: 10.1111/j.1365-246X.1984. tb05017.x.

de Bruin C, Wapenaar C and Berkhout A 1990 Angledependent reflectivity by means of prestack migration; Geophysics 55 1223-1234.

Du X, Bancroft J C and Lines L R 2007 Anisotropic reverse time migration for tilted TI media; Geophys. Prospect. $\mathbf{5 5}$ 853-869.

Duveneck E and Bakker P M 2011 Stable P-wave modeling for reverse-time migration in tilted TI media; Geophysics 76 S65-S75, doi: 10.1190/1.3533964.

Etgen J, Gray S H and Zhang Y 2009 An overview of depth imaging in exploration geophysics; Geophysics $\mathbf{7 4}$ WCA5-WCA17, doi: 10.1190/1.3223188.

Ferguson R and Margrave G F 1998 Depth migration in TI media by non-stationary phase shift; 68th Annual International Meeting, SEG, Expanded Abstracts 1831-1834.

Fletcher R P, Du X and Fowler P J 2009 Reverse time migration in tilted transversely isotropic (TTI) media; Geophysics 74 WCA179-WCA187.

Fowler P J, Du X and Fletcher R P 2010 Coupled equations for reverse time migration in transversely isotropic media; Geophysics 75 S11-S22.

Gray S H 2005 Gaussian beam migration of common-shot records; Geophysics 70 S71-S77, doi: 10.1190/1.1988186.

Gray S H and Bleistein N 2009 True-amplitude Gaussianbeam migration; Geophysics 74 S11-S23, doi: 10.1190/1. 3052116.

Han J G and Wang Y 2015 Gaussian beam prestack depth migration in heterogeneous transversely isotropic media; Explor. Geophys. 46 153-158.

Han J G, Wang Y and Lu J 2013 Multi-component Gaussian beam prestack depth migration; J. Geophys. Eng. 10 055008, doi: 10.1088/1742-2132/10/5/055008.

Han J G, Wang Y, Han N, Xing Z T and Lu J 2014a Multiwave velocity analysis based on Gaussian beam prestack depth migration; Appl. Geophys. 11 186-196.

Han J G, Wang Y, Xing Z T and Lu J 2014b Gaussian beam prestack depth migration of converted wave in TI media; J. Appl. Geophys. 109 7-14.

Hanyga A 1986 Gaussian beams in anisotropic elastic media; Geophys. J. Roy. Astr. Soc. 85 473-503.

Hill N R 1990 Gaussian beam migration; Geophysics $5 \mathbf{5}$ 1416-1428, doi: 10.1190/1.1442788.

Hill N R 2001 Prestack Gaussian-beam depth migration; Geophysics 66 1240-1250, doi: 10.1190/1.1487071.

Koren Z, Ravve I, Ragoza E, Bartana A and Kosloff D 2008 Full-azimuth angle domain imaging; 78th Annual International Meeting, SEG, Expanded Abstracts, pp. 2221-2225.

Larner K and Cohen J K 1993 Migration error in transversely isotropic media with linear velocity variation in depth; Geophysics 58 1454-1467, doi: 10.1190/1.1443360.

Liu H W, Li B, Liu H, Tong X L, Liu Q, Wang X W and Liu W Q 2012 The issues of prestack reverse time migration and solutions with Graphic Processing Unit implementation; Geophys. Prospect. 60 906-918.

Operto M S, Xu S and Lambaré G 2000 Can we quantitatively image complex structures with rays? Geophysics 68 1065-1074, doi: 10.1190/1.1444814.

Pedersen Ø, Ursin B and Helgesen H K 2010 One-way wave-equation migration of compressional and converted waves in a VTI medium; Geophysics 75 S237-S248, doi: 10.1190/1.3509466. 
Popov M M, Semtchenok N M, Popov P M and Verdel A R 2010 Depth migration by the Gaussian beam summation method; Geophysics 75 S81-S93, doi: 10.1190/1.3361651.

Protasov M I 2015 2-D Gaussian beam imaging of multicomponent seismic data in anisotropic media; Geophys. J. Int. 203 2021-2031.

Rickett J E and Sava P C 2002 Offset and angle-domain common image-point gathers for shot-profile migration; Geophysics 67 883-889, doi: 10.1190/1.1484531.

Ristow D 1999 Migration of transversely isotropic media using implicit finite-difference operators; J. Seism. Explor. 8 39-55.

Rosales D A, Fomel S, Biondi B L and Sava P C 2008 Wave-equation angle-domain common-image gathers for converted waves; Geophysics 73 S17-S26, doi: 10.1190/1.2821193.

Rousseau V, Nicoletis L, Svay-Lucas J and Rakotoarisoa H $20003 D$ true amplitude migration by regularization in the angle domain; 62nd Meeting, European Association of Geoscientists and Engineers, Session: B0013.

Sava P and Vlad I 2011 Wide-azimuth angle gathers for wave-equation migration; Geophysics 76 S131-S141, doi: 10.1190/1.3560519.

Sava P and Fomel S 2003 Angle-domain common-image gathers by wavefield continuation methods; Geophysics 68 1065-1074, doi: 10.1190/1.1581078.

Sena A G and Toksoz M N 1993 Kirchhoff migration and velocity analysis for converted and non-converted waves in anisotropic media; Geophysics 58 265-276.

Thomsen L 1986 Weak elastic anisotropy; Geophysics 51 1954-1966, doi: 10.1190/1.1442051.

Tong F, Joe A D, Gary E M, Jeffrey L H and Samuel H G 1998 Anisotropic true-amplitude migration; 68th Annual International Meeting, SEG, Expanded Abstracts, pp. $1677-1679$.

Wang Z J 2002 Seismic anisotropy in sedimentary rocks. Part 2: Laboratory data; Geophysics 67 1423-1440, doi: 10.1190/1.1512743.
Wang Q, Wang Y, Guo S G, Xing Z T and Liu Z W 2015 The effect of shale properties on anisotropic brittleness criterion index from laboratory study; J. Geophys. Eng. 12 866-874.

Winterstein D F 1990 Velocity anisotropy terminology for geophysicists; Geophysics 55 1070-1088, doi: 10.1190/ 1.1442919.

$\mathrm{Xu} \mathrm{S}$, Chauris H and Noble M 2001 Common-angle migration: A strategy of imaging complex media; Geophysics 66 1877-1894, doi: 10.1190/1.1487131.

$\mathrm{Xu}$ S, Zhang Y and Tang B 2011 3D common-image gathers from reverse time migration; Geophysics 76 S77-S92, doi: $10.1190 / 1.3536527$.

Yan R and Xie X B 2012 An angle-domain imaging condition for elastic reverse time migration and its application to angle gather extraction; Geophysics 77 S105-S115, doi: 10.1190/GEO2011-0455.1.

Yue $\mathrm{Y}$ B, Li Z C, Zhang $\mathrm{P}$, Zhou X F and Qin N 2010 Prestack Gaussian beam depth migration under complex surface conditions; Appl. Geophys. 7 143-148, doi: $10.1007 / \mathrm{s} 11770-010-0238-0$.

Zhang Q S and McMechan G A 2011 Direct vector-field method to obtain angle-domain common-image gathers from isotropic acoustic and elastic reverse time migration; Geophysics 76 WB135-WB149, doi: 10.1190/GEO20100314.1.

Zhan G, Pestana R C and Stoffa P L 2012 Decoupled equations for reverse time migration in tilted transversely isotropic media; Geophysics 77 T37-T45.

Zhang Y, Zhang H Z and Zhang G Q 2011 A stable TTI reverse time migration and its implementation; Geophysics 76 WA3-WA11, doi: 10.1190/1.3554411.

Zhang Y, Xu S, Tang B, Bai B, Huang Y and Huang T 2010 Angle gathers from reverse-time migration; Lead. Edge 29 1364-1371, doi: 10.1190/1.3517308.

Zhu T F, Gray S H and Wang D L 2007 Prestack Gaussianbeam depth migration in anisotropic media; Geophysics 72 S133-S138, doi: 10.1190/1.2711423. 